\title{
CAMBIO CLIMÁTICO GLOBAL EN EL CONTEXTO DE LA ECORREGIÓN SUBANTÁRTICA DE MAGALLANES Y LA RESERVA DE BIÓSFERA CABO DE HORNOS
}

\author{
GLOBAL CLIMATE CHANGE IN THE ECO-REGION SUBANTARCTIC OF \\ MAGELLAN AND THE CAPE HORN BIOSPHERE RESERVE
}

Andrés Mansilla ${ }^{1,3,4}$, Jaime Ojeda ${ }^{2,3}$ \& Ricardo Rozzi ${ }^{1,3,4,5}$

\begin{abstract}
RESUMEN
El cambio climático global no tiene consecuencias ni causas homogéneas en las diversas regiones del planeta, y sus consecuencias no son sólo ecológicas, sino también económicas, políticas y éticas. En este contexto global, la ecorregión subantártica de Magallanes y la dimensión ética representan dos aspectos que requieren mayor consideración por la comunidad científica. La primera posee singularidades biológicas y culturales que contrastan con zonas subpolares del Hemisferio Norte y deben ser examinadas con mayor atención respecto a su valor para confrontar los desafíos del cambio global ambiental. La segunda requiere un examen detallado por al menos dos razones: a) las causas últimas del cambio climático global radican en un modo de relación establecido por la sociedad industrial con el mundo natural, que se ha globalizado y radicalizado a partir de mediados del siglo XX; b) la pregunta de cómo debemos actuar frente al cambio medioambiental global involucra decisiones éticas. En este marco de singularidades el "Coloquio Internacional de Cambio Climático en la Región de Magallanes y Antártica: Evidencia y Desafíos para el Futuro" organizado por la Universidad de Magallanes (UMAG) y el Instituto Antártico Chileno, en octubre 2009, abre nuevas oportunidades para la investigación socio-ecológica a largo plazo y el monitoreo del cambio ambiental global a escala planetaria. Con el fin de contribuir a la consolidación de estas iniciativas presentamos concisamente los atributos de la ecorregión subantártica de Magallanes y del programa de Conservación Biocultural Subantártica, coordinado por UMAG, el Instituto

1 Laboratorio de Macroalgas Antárticas y Subantárticas - LMAS, Departamento de Ciencias y Recursos Naturales, Universidad de Magallanes, Casilla 113-D, Punta Arenas, Chile. andres.mansilla@umag.cl.

2 Programa de Magíster en Ciencias con Mención en Manejo y Conservación de Recursos en Ambientes Subantárticos, Universidad de Magallanes, Casilla 113-D, Punta Arenas, Chile.

3 Parque Etnobotánico Omora, Centro Universitario Puerto Williams, Universidad de Magallanes, Chile.

4 Instituto de Ecología y Biodiversidad, Casilla 653, Santiago, Chile.

5 Sub-Antarctic Biocultural Conservation Program, Department of Philosophy and Religion Studies, University of North Texas, Denton TX, USA.
\end{abstract}


de Ecología y Biodiversidad y la Universidad de North Texas, que integra dimensiones ecológicas y éticas en los análisis de procesos socio-ecológicos a escalas locales, regionales, nacionales e internacionales.

Palabras clave: Ética Ambiental, Estudios Ecológicos al Largo Plazo, Chile.

\section{ABSTRACT}

The consequences and causes of global climate change are not homogeneous in the diverse regions of the planet, and its impacts are not only ecological, but also economic, political, and ethical. In this context, the sub-Antarctic Magellanic ecoregion and the ethical dimensions represent two aspects that require more attention from the scientific community. The first has biological and cultural singularities that contrast with subpolar regions of the Northern Hemisphere, and need to be given greater consideration to effectively confront the challenges of global environmental change. The second requires more in depth studies for at least two reasons: a) the ultimate causes of global climate change derive from the type of relationship established by industrial society with the natural world, a type of relationship that has become increasingly prevailing since the mid-20 $0^{\text {th }}$ century; b) the question about we should act in the face of global change involves ethical questions. In this context, the International Colloquium on Global Climate Change in the Region of Magallanes and Antarctica: Evidences and Future Challenges, organized by the University of Magallanes and Chilean Antarctic Institute in October 2009, opens new opportunities for long-term socio-ecological research and monitoring of global environmental change at a planetary scale. To contribute to the consolidation $\mathrm{n}$ of these initiatives we concisely present the main attributes of Magellanic sub-Antarctic ecoregion, and of the Sub-Antarctic Biocultural Conservation Program coordinated by UMAG, the Institute of Ecology and Biodiversity and the University of North Texas, which integrates ecological and ethical dimensions in the analyses of socio-ecological processes at local, regional, national, and international scales.

Key words: Environmental ethics, Long-term Ecological Research. Chile.

\section{INTRODUCCIÓN}

El cambio climático global es un fenómeno respecto al cual la comunidad científica ha alcanzado al menos cinco conclusiones demostrables (Gleick et al. 2010): i) el planeta se está calentando por el aumento de las concentraciones de gases invernaderos; ii) el aumento de las concentraciones de los gases invernaderos durante el último siglo se debe principalmente a actividades humanas, especialmente la deforestación y la combustión de combustibles fósiles; iii) las causas naturales siempre han jugado un papel central en los cambios climáticos del planeta, sin embargo, hoy son sobrepasadas por los cambios inducidos por el hombre. iv) El calentamiento del planeta está generando otras alteraciones climáticas a una velocidad sin precedentes en tiempos históricos, y sus consecuencias incluirán aumentos en el nivel del mar, alteraciones en los ciclos hidrológicos y el aumento de las concentraciones $\mathrm{CO}_{2}$ provocará una acidificación de los océanos; v) la combinación de estos complejos cambios climáticos amenaza las comunidades y ciudades costeras, los suministros de alimentos y agua, la integridad de ecosistemas marinos, dulceacuícolas, forestales, de alta montaña y otros. El cambio climático global no tiene consecuencias ni causas homogéneas en las diversas regiones del planeta, y sus consecuencias no son solo ecológicas, sino también económicas, políticas y éticas (Chapin et al. 2010). En este contexto global, la ecorregión subantártica de Magallanes y la dimensión ética representan dos aspectos que requieren mayor consideración por la comunidad científica (Rozzi et al. 2012). La primera posee singularidades biológicas y culturales que contrastan con zonas subpolares del Hemisferio Norte y deben ser examinadas con mayor atención respecto a su valor para confrontar los desafíos del cambio global ambiental. La segunda requiere un examen detallado por al menos dos razones: a) las causas últimas del cambio climático 
global radican en un modo de relación establecido por la sociedad industrial con el mundo natural, que se ha globalizado y radicalizado a partir de mediados del siglo XX (White 1967, Rozzi 2001); b) la pregunta de cómo debemos actuar frente al cambio medioambiental global involucra decisiones éticas (Rozzi et al. 2010). Las ciencias ecológicas contribuyen a caracterizar las causas próximas de la crisis ambiental global, y ayudan a definir qué podemos hacer, pero definir qué debemos hacer involucra esencialmente una respuesta ética sujeta a procesos de conflicto y deliberación entre diversos actores con intereses y valores contrastantes (Rozzi et al. 2010). En el marco del "Coloquio Internacional de Cambio Climático en la Región de Magallanes y Antártica: Evidencias y Desafíos para el Futuro" presentamos concisamente los atributos de la ecorregión subantártica de Magallanes y del programa de Conservación Biocultural Subantártica que integra dimensiones ecológicas y éticas en los análisis de procesos socio-ecológicos a escalas locales, regionales, nacionales e internacionales.

\section{Ecorregión subantártica de Magallanes}

La ecorregión subantártica de Magallanes ha estado sujeta a la influencia de los avance y retroceso de los hielos ocurridos durante el cuaternario (Silva \& Calvete 2002). Gran parte de su actual sistema de canales estuvo cubierto por hielo hasta el Ultimo Máximo Glacial (UMG), entre 23.000 y 19.000 años A.P (Hulton et al 2002). El proceso de desglaciación desde el UMG ha permitido la recolonización de diversas formas de vida (Hulton et al. 2002), generando una ecorregión que ha sido identificada recientemente como una las 24 áreas mejor conservadas del planeta (Mittermeier et al. 2002, Mittermeier et al. 2003). Los criterios de esta identificación consideran que (i) más del 70\% de su cobertura vegetacional nativa se conserva, (ii) incluye una vasta área geográfica de más de $10.000 \mathrm{~km}^{2}$ de hábitats nativos no fragmentados, y (iii) posee una de las menores densidades poblacionales en zonas templadas a nivel mundial $\left(0,14\right.$ habitantes $\left./ \mathrm{km}^{2}\right)$. Entre las singularidades de la diversidad biológica y cultural de los ecosistemas terrestres y marinos de la ecorregión subantártica de Magallanes, al menos diez atributos sobresalen a nivel regional y mundial (Rozzi et al. 2012): i) Inlcuye los bosques más australes del planeta, que se extienden hasta el Cabo de Hornos (56ㅇ), diez grados de latitud más al sur que los bosques templados de Nueva Zelanda y Australia, y representan los ecosistemas forestales más cercanos a la Antártica (Silander 2000, Rozzi et al. 2006).

ii) Representan el límite extremo de latitud sur para la distribución de numerosos grupos taxonómicos a nivel de especies, géneros, familias e incluso órdenes y clases (Rozzi et al. 2007).

iii) Las barreras topográficas y climáticas generadas por la cordillera de los Andes, el desierto de Atacama y la vasta extensión oceánica aíslan al bioma de los bosques templados del sudoeste de Sudamérica. Este aislamiento geográfico ha generado niveles de endemismo notablemente altos. Entre las plantas vasculares, cerca de un 90\% de las especies leñosas son endémicas (Arroyo et al. 1996), y entre las briófitas los niveles de endemismo superan al 50\% de las especies (Villagrán et al. 2005), En los fiordos también se registran altos niveles de diferenciación genética, y estos ecosistemas costeros constituyen laboratorio natural único para el estudio de procesos evolutivos (e.g. González-Wevar et al. 2011).

iv) El margen sudoeste de Sudamérica alberga grupos taxonómicos de flora, como musgos y hepáticas, y de fauna, como moluscos, que aumentan su riqueza de especies hacia latitudes más altas (e.g. musgos, hepáticas y moluscos) y alcanzan una diversidad máxima en la región subantártica de Magallanes (Valdovinos et al. 2003, Rozzi et al. 2008). En los ecosistemas costeros sobresalen los grandes "bosques submarinos" de algas pardas, conocidos internacionalmente como "kelps", que alcanzan una de las mayores extensiones y biomasas de estas algas a nivel mundial, y ofrecen un hábitat crítico para muchas especies de invertebrados y vertebrados (Mansilla \& Avila 2011).

v) El bioma de los bosques templados de Sudamérica alberga las mayores superficies de bosques templados del Hemisferio Sur (Bryant et al. 1997)

vi) El bioma de los bosques templados de Sudamérica alberga las mayores superficies de humedales templados del Hemisferio Sur (Keddy et al. 2009)

vii) En la ecoregion subantártica se han registrado las aguas de lluvia y cursos dulceacuícolas más limpios del planeta, al estar más al sur de las corrientes de vientos que transportan contaminan- 
tes industriales y recibir sus lluvias directamente de frentes que se originan en el Océano Pacífico Sur, en esta ecorregión se han medido las concentraciones más bajas de nitratos registradas en aguas de lluvia a fines del siglo XX (Likens 1991, Hedin et al. 1995, Weathers et al. 2000).

viii) En la ecoregion subantártica se encuentran se encuentran grandes áreas de hielos continentales que abracan $4.200 \mathrm{~km}^{2}$ y $13.000 \mathrm{~km}^{2}$ en los Campos de Hielo Patagónico Norte y Sur, respectivamente, y $2300 \mathrm{~km}^{2}$ en los extensos sistemas glaciares de Cordillera Darwin y archipiélagos aledaños (Porter \& Santana 2003). En conjunto, estos glaciares representan (a) las mayores masas de hielo fuera de la Antártica en el Hemisferio Sur, (b) una vasta reserva de agua dulce; (c) depósitos con registros únicos de eventos climáticos pasados en latitudes altas del Hemisferio Sur, y (d) son más sensible a cambios térmicos globales que sus contrapartes latitudinales en Alaska (Rignot et al. 2003)

ix) La historia de ciclos glaciares documentados en registros palinológicos conservados en turberas y humedales de la ecorregión subantártica de Magallanes provee un sitio único para estudiar eventos pasados y comprender posibles escenarios futuros de cambios climáticos (Villa-Martínez and Moreno 2007). x) La ecorregión subantártica ha sido habitada por etnias que han cultivado una estrecha relación con sus hábitats y desarrollado un refinado conocimiento ecológico, que incluyen a los pueblos kaweshkar, selknam y a la etnia más austral del planeta, el pueblo yagán (Hidalgo et al. 1996, Martinic 2003, 2006).

El conjunto de características biológicas, geográficas y culturales caracterizan a la ecorregión subantártica de Magallanes como una zona única para el estudio de cambio climático global (Fig. 1). Esta ecorregión no posee réplica en el planeta, y sus singularidades constituyeron el fundamento para la creación el año 2005, de la Reserva de Biosfera Cabo de Hornos (RBCH) que presenta una extensión de 4.884.274 ha de ecosistema marinos y terrestres (Rozzi et al. 2007). El proceso de creación de la $\mathrm{RBCH}$ fue liderado por el equipo científico, interdisciplinario del "Parque Etnobotánico Omora" que hoy integra la Red Chilena de Sitios de Estudios Socio-Ecológicos a Largo Plazo (SESELP) (Fig. 1).

Para potenciar la investigación de las singularidades de la diversidad subantártica biológica y cultural y sus interrelaciones, como también, sus vulnerabilidades al cambio ambiental global, en la Reserva de Biosfera Cabo de Hornos, investigadores de la Universidad de Magallanes, del Instituto de

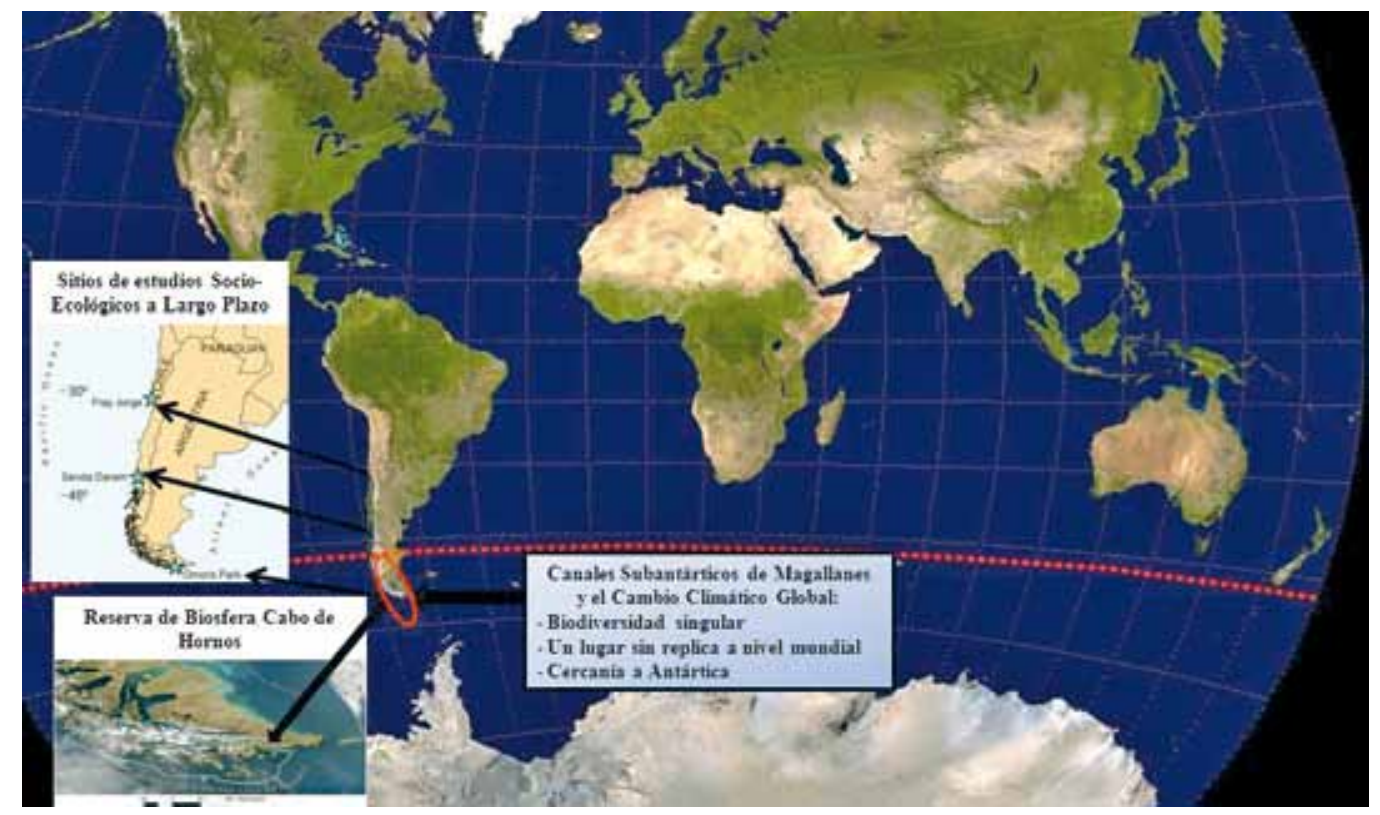

Fig. 1. Los canales subantárticos de Magallanes son un lugar especial para los estudios del Cambio Climático Global por sus singularidades biológicas y geográficas. Herramientas de investigación, educación y conservación de la biodiversidad son la Reserva de Biosfera Cabo de Hornos y la red Chilena de Estudios Socio Ecológicos a Largo Plazo. 
Ecología y Biodiversidad y de la University of North Texas - USA, reforzaron el Parque Omora como un centro de estudios a largo plazo en el extremo austral de América.

Parque Etnobotánico Omora: Centro de Estudios Subantárticos en la Reserva de Biosfera Cabo de Hornos

En colaboración con educadores, políticos, filósofos y la comunidad en general, el equipo científico del Parque Omora ha investigado y dado a conocer tanto las singularidades bioculturales como las amenazas a las que está sujeta hoy la diversidad biocultural de la ecorregión subantártica de Magallanes. Estas amenazas incluyen crecientes presiones de desarrollo, tales como el turismo masivo, acuicultura de especies exóticas principalmente la salmonicultura, sobrepastoreo, especies exóticas invasivas, homogeneización cultural, debilitamiento de la capa de ozono atmosférico con un incremento de la radiación UV, retrocesos de glaciares y otros efectos del cambio climático global. Para organizar sus actividades de investigación, educación y conservación en colaboración con diversas instituciones, el Parque Omora ha definido una estructura que en tres escalas interdependientes y sinérgicas: (A) local-regional, (B) nacional, y (C) internacional (Rozzi et al. 2010).

A) Escala local-regional: el Parque Etnobotánico Omora tiene una extensión de aproximadamente 1.000 ha, y corresponde a una concesión gratuita por 25 años del Ministerio de Bienes Nacionales a la Universidad de Magallanes y a la Fundación Omora. Estas instituciones co-ejecutan sus programas en colaboración con el Instituto de Ecología y Biodiversidad (IEB). Para el trabajo a escala local-regional, el Parque Omora cumple el papel de centro científico interdisciplinario en la Reserva de Biosfera Cabo de Hornos (RBCH). Este trabajo se desarrolla en estrecha asociación con el Programa de Magister en Ciencias Mención en "Conservación y Manejo de Recursos Naturales en Ambientes Subantárticos" de la Universidad de Magallanes, que constituye el primer programa de Magister acreditado en la Región de Magallanes y Antártica Chilena (Comisión Nacional de Acreditación, 2011¹).

1 CNA-CHILE, 2011. Comisión Nacional de Acreditación. $<$ En Línea> URL: www.cnachile.cl
B) Escala nacional: Red Chilena de Sitios de Estudios Socio-Ecológicos a Largo Plazo En el año 2008 el Parque Omora cofundó la primera Red Chilena SESELP con financiamiento del Programa de Financiamiento Basal de CONICYT y la Iniciativa Científica Milenio de MIDEPLAN. La meta inicial de la red (2008-2011) ha sido consolidar los programas de investigación y educación que actualmente existen entre los tres sitios fundadores: Sitio Experimental Fray Jorge (30 S) (Gutiérrez et al. 2010), la Estación Biológica Senda Darwin $\left(42^{\circ} \mathrm{S}\right)$ (Carmona et al. 2010) y el Parque Omora (55 S). La red tiene como misión fundamental desarrollar investigación a largo plazo, reforzar e implementar cursos de campos nacionales, internacionales e interdisciplinarios. La Red Chilena SESELP coordinada por el IEB, ha establecido un registro de variables biofísicas para caracterizar procesos ecosistémicos y climáticos con metodologías comparables entre los tres sitios, y con la Red Internacional de Sitios de Estudios Ecológicos a Largo Plazo (ILTER, International Long-Term Ecological Research por su sigla en inglés). Estos estudios proveen líneas de base que pueden ser utilizadas para realizar experimentos a largo plazo y evaluar las respuestas de la biota y los ecosistemas frente al cambio climático global en un gradiente latitudinal en el sudoeste de Sudamérica.

C) Escala internacional: El Programa de Conservación Biocultural Subantártica coordinado por la Universidad de Magallanes (UMAG), el Instituto de Ecología y Biodiversidad (IEB) y la Universidad de North Texas (UNT) han integrado tres dominios de acción: investigación, educación y conservación (Rozzi et al. 2006). Algunos desafíos del programa es que la investigación ecológica tenga una relevancia social en el contexto de cambio ambiental global. Logro que se concreta mediante la articulación de programas de investigación a escalas locales y nacionales con escalas globales, y la integración de las ciencias ecológicas y la ética ambiental. Para lograr estos dos objetivos, la Universidad de North Texas (UNT) se ha constituido un colaborador ideal por dos razones: i) alberga el Centro de Filosofía Ambiental (Center for Environmental Philosophy, CEP, http://www.cep.unt.edu) que en 1978 fundó la revista Ética Ambiental (Environmental Ethics), la primera a nivel mundial en la joven subdisciplina de la ética ambiental. Estas colaboraciones han pro- 
movido un diálogo interdisciplinario entre ecólogos, filósofos, artistas y otros pensadores ambientales latinoamericanos y estadounidenses, y desarrollar la metodología de filosofía ambiental de campo que permite integrar las ciencias ecológicas y la ética ambiental en programas de postgrado y ecoturismo que procuran contribuir a la conservación biocultural en la ecorregión subantártica de Magallanes (Rozzi et al. 2012).

Coloquio Internacional de Cambio Climático en la Región de Magallanes y Antártica

Con el propósito de abordar las singularidades de la diversidad biológica y cultural de los ecorregión subantártica de Magallanes, y en particular de la Reserva de Biosfera Cabo de Hornos, se han creado redes y formas de colaboración que generan puentes esenciales en la complementariedad del trabajo a escalas local, regional y global. A través de las colaboraciones interdisciplinarias, el cambio climático o el cambio medioambiental se trasforman además no sólo en un problema ecológico sino también ético y cultural. Para ello, se han convocado instancias de interacción y discusión in situ tal como el "Coloquio Internacional de Cambio Climático en la Región de Magallanes y Antártica: Evidencias y Desafíos para el Futuro", que en octubre 2009 ha sido organizado por la Universidad de Magallanes y el Instituto Antártico Chileno. Estas instancias han potenciado el estudio y discusión colaborativa acerca de la singular diversidad biocultural subantártica a partir delo trabajo compartido entre investigadores nacionales $e$ internacionales. La Sesión, denominada Diversidad y Cambio Climático consideró la participación de destacados científicos, quienes presentaron perspectivas complementarias que incluyeron:

A) Dr. Fernando Santibáñez, Facultad de Agronomía Universidad de Chile: Dimensionando el impacto de los cambios climáticos sobre los ecosistemas naturales y agrícolas

B) Dra. Laura Meza, Oficina Regional para América Latina y el Caribe (RLC) FAO: Modelo de Gestión de los Riesgos a Eventos Climáticos Extremos con Impacto en la Agricultura en la Región de Magallanes
C) Dra. Mary Kalin, Instituto de Ecología y Biodiversidad Universidad de Chile: Cambios en los principales tipos de vegetación en Chile pronosticados bajo cambio climático - modelos, incertezas y adaptaciones a la investigación para un mundo de biodiversidad dinámica

D) Dra. Carina Lange, Centro FONDAPCOPAS, Universidad de Concepción, Chile: Investigaciones destacadas de los fiordos chilenos: columna de agua y sedimentos

Agradecemos la participación de estos y el conjunto de investigadores convocados en el Coloquio Internacional de Cambio Climático en la Región de Magallanes y Antártica, quienes con iniciativas como estas refuerzan el trabajo colaborativo de los científicos Magallánicos, y los marcos conceptuales para comprender el valor de los ecosistemas únicos en el planeta, como aquellos albergados por la ecoreregión subantártica de Magallanes.

\section{AGRADECIMIENTOS}

Este trabajo ha contado con el apoyo de la Iniciativa Científica Milenio (ICM, P05-002), del Programa de Financiamiento Basal de CONICYT (PFB-23) y del Programa de Conservación Biocultural Subantártica (Universidad de Magallanes, Instituto de Ecología y Biodiversidad de Chile y University of North Texas (http://www.chile.unt.edu).

\section{LITERATURA CITADA}

Armesto, J.J., R. Rozzi, C. Smith-Ramírez \& M.T.K. Arroyo 1998. Conservation targets in South American temperate forests. Science, 282: 1271-1272

Arroyo, M.T.K., L. Cavieres, A. Peñaloza, M. Riveros \& A.M. Faggi 1996. Relaciones fitogeográficas y patrones regionales de riqueza de especies en la flora del bosque lluvioso templado de Sudamérica. En: Armesto J.J., Villagrán C., Kalin Arroyo M., eds. pp. 71-92 Ecología de los Bosques Nativos de Chile. Editorial Universitaria, Santiago de Chile.

Bryant, D., D. Nielsen \& L. Tangley 1997. The Last Frontier Forests: Ecosystems and Economies 
on the Edge. World Resources Institute, Washington, D.C.

Carmona, M.R., J.C. Aravena, M.A. BustamanteSanchez, J.L. Celis-Diez, A. Charrier, I. A. Díaz, J. Díaz-Forestier, M. F. Díaz, A. Gaxiola, A. G. Gutiérrez, C. Hernandez-Pellicer, S. Ippi, R. Jaña-Prado, P. Jara-Arancio, J. Jimenez, D. Manuschevich, P. Necochea, M. NuñezAvila, C. Papic, C. Pérez, F. Pérez, S. Reid, L. Rojas, B. Salgado, C. Smith-Ramírez, A. Troncoso, R. A. Vásquez, M. F. Willson, R. Rozzi \& J.J. Armesto 2010. Estación Biológica Senda Darwin: Investigación ecológica de largo plazo en la interfase ciencia-sociedad. Revista Chilena de Historia Natural, 83: 113-142

Chapin, F.S. III, M. Power, S.T. Pickett, D. Carter, R. Jackson \& C. Duke 2010. Earth Stewardship: A framework to transform the trajectory of society's relationship to the biosphere. SBE White Paper 9. Ecological Society of America. (29 March 2011; www. esa.org/earthstewardship/files/SBEWhitePaper9_29\%20ESA.pdf).

Gleick, P.H., R.M. Adams, R.M. Amasino, E. Anders, D.J. Anderson, et al. 2010. Climate change and the integrity of science. Science, 328: 689-690.

González-Wevar, C.A., T. Nakano, J.I. Cañete, E. Poulin 2011. Concerted genetic, morphological and ecological diversification in Nacella limpets in the Magellanic Province. Molecular Ecology, 20: 1936-1951

Gutiérrez, J.R., P.L. Meserve, D.A. Kelt, A. Engilis J.R., M.A. Previtali, W.B. Milstead \& F.M. Jaksic 2010. Long-term research in Bosque Fray Jorge National Park: Twenty years studying the role of biotic and abiotic factors in a Chilean semiarid scrubland. Revista Chilena de Historia Natural, 83: 69-98

Hedin, L.O., J.J. Armesto, \& A.H. Johnson 1995. Patterns of nutrient from unpolluted, old-growth temperate forest: Evaluation of biogeochemical theory. Ecology, 76: 493-509

Hidalgo, J., V. Schiappacasse, H. Niemeyer, C. Aldunate \& P. Mege 1996. Etnografía, Sociedades Indígenas Contemporáneas y Su Ideología. Editorial Andrés Bello, Santiago, Chile, pp. 303.
Hulton, N.R.J., R.S. Purves, R.D. Mcculloch, D.E. Sugden \& M.J. Bentley 2002. The Last Glacial Maximum and deglaciation in southern South America. Quaternary Science Reviews, 21: 233-241

Keddy, P.A., L.H. Fraser, A.I. Solomeshch, W.J. Junk, D.R. Campbell, M.T.K. Arroyo \& C.J.R. Alho 2009. Wet and wonderful: The world's largest wetlands are conservation priorities. Bioscience, 59: 39-51

Likens, GE. 1991. Consequences of long-term human impacts on ecosystems. Revista Chilena de Historia Natural, 64: 597-614

Mansilla, A. \& M. Avila 2011. Using Macrocystis pyrifera (L.) C. Agardh from southern Chile as a source of applied biological compounds. Bras Farmacogn 21: 262-267

Martinic, M. 2003. Archipiélago Patagónico. La Última Frontera. Ediciones de la Universidad de Magallanes, Punta Arenas, Chile, pp. 297

Martinic, M. 2006. Crónica de las Tierras del Sur del Canal Beagle. Ediciones Hotel Lakutaia. Punta Arenas, Chile, pp. 279.

Mittermeier, R.A., C.G. Mittermeier, T.M. Brooks, J.D. Pilgrim, W.R. Konstant, G.A.B. Da Fonseca \& C. Kormos 2003. Wilderness and biodiversity conservation. Proceedings of the National Academy of Sciences, 100: 10309-10313

Mittermeier, R.A., C.G. Mittermeier, P. Robles-Gil, J.D. Pilgrim, G.A.B. Da Fonseca, T.M. Brook \& W.R. Konstant 2002. Wilderness: Earth's Last Wild Places. CEMEX-Conservation International, Washington DC., pp. 576

Porter, C. \& A. Santana 2003. Rápido retroceso, en el siglo 20, del ventisquero Marinelli en el campo de hielo de la Cordillera Darwin. Anales del Instituto de la Patagonia, 31: 17-26

Rignot, E., A. Rivera \& G. Casassa 2003. Contribution of the Patagonia Icefields of South America to Sea Level Rise. Science, 302: 434-437

Rivera, A \& G. Casassa 2004. Ice elevation, area and frontal changes of glaciers from Torres del Paine National Park, Southern Patagonia Icefield. Arctic, antarctic and alpine research, 36: 379-389

Rozzi, R., F. Massardo, J. Silander, O. Dollenz, B. Connolly, C.B. Anderson \& N. Tuner 2003. 
Árboles Nativos y Exóticos en las plazas de Magallanes. Anales del Instituto de la Patagonia, 31:27-42

Rozzi, R., F. Massardo, C. Anderson, K. Heidinger \& J. Silander Jr. 2006. Ten principles for biocultural conservation at the southern tip of the Americas: The approach of the Omora Ethnobotanical Park. Ecology \& Society, 11: 43. (12 April 2011; www.ecologyandsociety. org/vol11/iss1/art43/).

Rozzi, R., F. Massardo, A. Mansilla, C.B. Anderson, A. Berghofer, M.O. Mansilla, M.R. Gallardo, J. Plana, U. Berghofer, X. Arango, S. Russell, P. Araya \& E. Barros 2007. La Reserva de Biosfera Cabo de Hornos: un desafío para la conservación de la biodiversidad e implementación del desarrollo sustentable en el extremo austral de América. Anales del Instituto de la Patagonia, 35: 55-70

Rozzi, R., J.J. Armesto, B. Goffinet, W. Buck, F. Massardo, J.A. Silander Jr, M.T.K. Arroyo, S. Russell, C.B. Anderson, L.A. Cavieres \& J.B. Callicott 2008. Changing biodiversity conservation lenses: insights from the subAntarctic non-vascular flora of southern South America. Frontiers in Ecology and the environment, 6: 131-137

Rozzi, R., C.B. Anderson, J.C. Pizarro, F. Massardo, Y. Medina, A. Mansilla, J.K. Kenedyy, J. Ojeda, T. Contador, V. Morales, K. Moses, A. Poole, J.J. Armesto, M.T. Kalin 2010. Filosofía ambiental de campo y conservación biocultural en el Parque Etnobotánico Omora: Aproximaciones metodológicas para ampliar los modos de integrar el componente social ("S") en Sitios de Estudios Socio-Ecológicos a Largo Plazo (SESELP). Revista Chilena de Historia Natural, 83: 27-68
Rozzi, R., J.J. Armesto, J. Gutiérrez, F. Massardo, G. Likens, C.B. Anderson, A. Poole, K. Moses, G. Hargrove, A. Mansilla, J.H. Kennedy, M. Willson, K. Jax, C. Jones, J.B. Callicott \& M.T. Kalin 2012. Integrating ecology and environmental ethics: Earth stewardship in the southern end of the Americas. BioScience, 62 (3): 226-236

Silander, J.A. Jr. 2000. Temperate forests: plant species biodiversity and conservation. In (S.A. Levin ed.) Encyclopedia of Biodiversity. Academic Press, New York, pp. 607-626.

Silva, N. \& C. Calvete 2002. Características oceanográficas físicas y químicas de canales australes chilenos entre el golfo de penas y el Estrecho de Magallanes (Crucero CIMAR-FIORDOS 2). Ciencia y tecnología del mar, 25: 23-88

Valdovinos, C., S.A. Navarrete \& P.A. Marquet 2003. Mollusk species diversity in the Southeastern Pacific: Why are there more species towards the pole. Ecography, 26: 139-144

Villagran, C., G. Hazle \& F. Barrera 2005. Hepáticas y antocerotes del archipiélago de Chile. Corporación de Amigos, Museo Nacional de Historia Natural, Santiago, Chile, pp. 160.

Villa-Martínez, R. \& P. Moreno 2007. Pollen evidence for variations in the southern margin of the westerly winds in SW Patagonia over the last 12,600 years. Quaternary Research 68: 400-409

Weathers, K.C., G.M. Lovett, G.E. Likens \& N.F.M. Caraco 2000. Cloudwater inputs of nitrogen to forest ecosystems in southern Chile: Forms, fluxes, and sources. Ecosystems, 3: 590-595

White, L. 1967. Raíces históricas de nuestra crisis ecológica. En: Edición Especial: Ética Ambiental (Rozzi, R., P. Villaroel \& F. Massardo, eds), Revista Ambiente y Desarrollo 23: 78-86 